\title{
20.
}

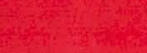

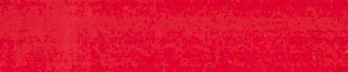

s. 60 istes

-ixy
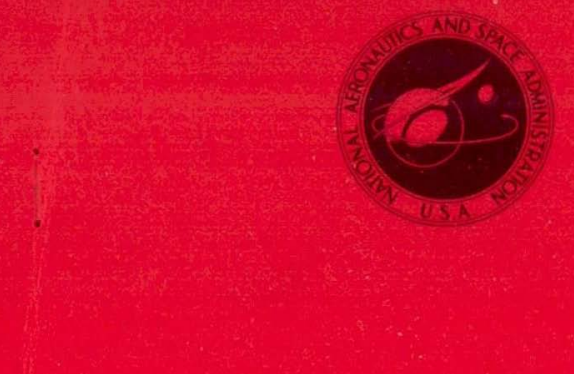

\section{NASA RP-40}

3y.

TECHNICAL REPRINT

NATIONAL AERONAUTICS AND SPACE ADMINISTRATION WASHINGTON, D.C. 
Trapped Particles from High-Altitude Nuclear Explosions

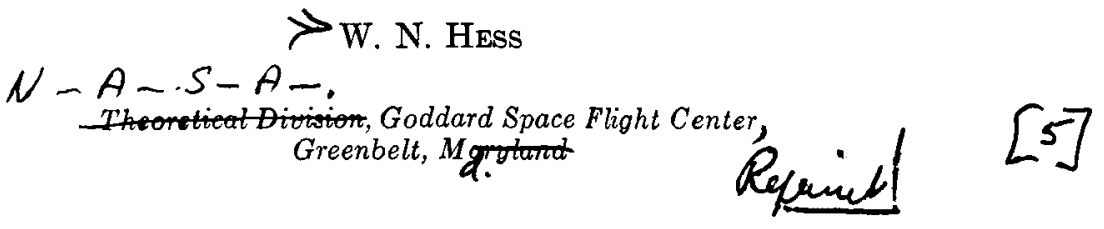

A series of invited papers was given on the artificial radiation belt containing a substantial amount of new information. The first large amount of data from the Explorer 15 satellite was released.

Walter Brown of Bell Telephone Laboratories and Carl McIlwain of the University of California, San Diego, discussed their experiments on this new satellite, which was launched specifically to study the trapped particles. Measurements of electrons and protons in several different energy regions were reported. Time histories of particles were shown, and a discussion of the results of the USSR high-altitude explosions on October 28 and November 1, 1962, was presented. The October 28 explosion produced a belt of electrons ranging from $L=1.8$ up to about $L=3$. Near $L=1.8$ the electrons had essentially a fission energy spectrum, but at higher $L$ values the spectral became steadily softer. This belt of particles decayed with lifetimes of the order of one week, in rough agreement with the earlier measurements from Telstar shortly after July 9. The time histories for large $L$ values show significant changes associated with magnetic disturbances.

Ray Smith of Lockheed discussed measurements of several DOD satellites and showed information substantiating theories that at lower altitudes the particle flux is not dependent on $B$ and $L$ only, but it also changes with longitude due to the strong scattering in the region of the South Atlantic magnetic anomaly.

James A. Van Allen of Iowa discussed measurements from the Injun satellites and showed docay characteristics of the trapped electrons in the region $L=1.2$ to 1.3 . These curves indicate long lifetimes for the electrons and show wat the decay at different points on one field line is now quite similar. This agrees with the theory that the decay rates at all points along the line should be dominated by the scattering rate at the equator after scattering equilibrium has been obtained. Van Allen's new data give a total flux of $1.3 \times 10^{25}$ fission electrons trapped 10 hours after the Starfish explosion. This is not the total population of new electrons; it only represents those having a fission electron spectrum.

Stirling A. Colgate of Livermore Radiation Laboratory and W. N. Hess of Goddard Space Flight Center discussed problems concerning the injection of electrons from the explosion. Colgate showed that as a result of encountering a cubic scale height of air, the debris from Starfish should slow down, and most of it should follow a field line and be deposited at the opposite conjugate point. This agrees with aircraft measurements of the gamma ray in the southern conjugated area. Colgate indicated that several per cent of the debris could go to substantially higher altitudes. Hess discussed the problems of the spatial distribution of the electrons, resulting from the decay of the debris, and the energy spectrum of the electrons. He indicated that two processes might modify the electrons' energy spectrum: decay of fission fragments sometime after the fission event will produce lower-energy electrons, and electrons may be slowed down by Fermi deceleration on the walls of an expanding bubble in the field. Such processes may explain the spectral changes observed from the Soviet October 28 explosion. 